\title{
A Clinical Study of Myopia
}

\section{Dr.Mande Vijaya Leela M.S(opthal), Dr.D. Bhima Sankar Babu M.S(opthal) \\ Asst.professor of ophthalmology Osmania medical college/sarojini devi eye hospital}

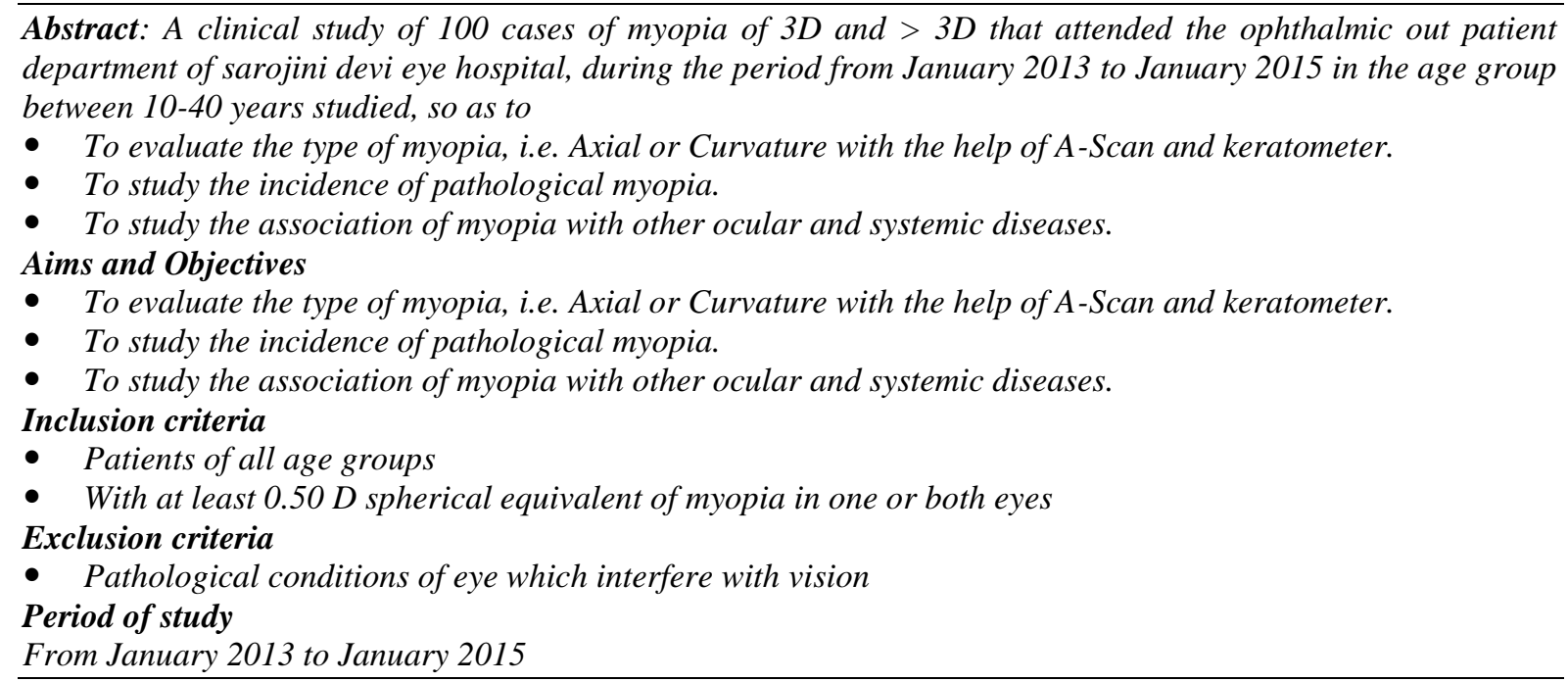

- Myopia studies are notoriously difficult to carry out. Studies on myopia have taken researchers in the past on a roller-coaster ride. The most important reason for this is the very variable nature of myopia. The very variable nature of myopia raises the question of whether an apple is being compared with another apple or with the same apple.

- Because myopia progression is linear in its early stage until the early teenage years, it is possible to observe what happens to the linear progression upon intervention. Two children of the same race, sex, age and degree of myopia, progress very differently and hence, various designs for myopia studies haven been adopted in the past, with a view to achieving good baseline comparability, reliability and repeatability

- To study the clinical aspects of Myopia with particular reference to demographic variables and social; factors, in the patients attending to Sarojini devi eye hospital's Ophthalmic Out Patient Department.

\section{Purpose of the study:}

- To investigate risk factors for the development of myopia.

- Myopia Clinical research trials and Myopia health trials happen in many localities throughout the World. A clinical trial or clinical study is a research project with human volunteer subjects. The aim of the studies / projects is to resolve certain human healthcare questions. The present study is also one such clinical study of Myopia, to examine and to better understand various clinical aspects of myopia with special focus on demographic variables.

The out come of this study would give clinically meaningful information with regards to preventative, diagnostic, and therapeutic aspects so that the currently available modalities can be utilized much more meaningfully for the benefit of patient

\section{Methodology}

- This study comprises a cross sectional study of patients with Myopia who attended Ophthalmic O.P of sarojini devi eye hospital, hyderabad between 2014and 2015. Each subject was interviewed, a brief history of the age of onset of defective vision, involvement of other members of the family, the place of residence, and whether the parents' marriage was consanguineous, was recorded.

- Evaluation included visual assessment, and anterior and posterior segment examinations

- For the study, 100 subjects underwent an extensive ophthalmologic screening examination, including measurements of visual acuity and the visual field and fundus photography. The causes of cataract or visual impairment are determined using all screening information and medical records 
- The results thus obtained are analyzed, and discussed in comparison with the existing studies in the literature. SPSS Version 10.1 software has been used for statistical analysis. Descriptive statistics are computed for baseline demographic, clinical and laboratory features according to etiology. Frequency tables were generated to reflect the etiology of paediatric cataract and all the values are determined at 5\% significance level. Contingency tables were analyzed through chisquared statistics

\section{Discussion}

- Various forms of myopia have been described by their clinical appearance

- Simple myopia is more common than other types of myopia and is characterized by an eye that is too long for its optical power (which is determined by the cornea and crystalline lens) or optically too powerful for its axial length. Both genetic and environmental factors, particularly significant amounts of near work, are thought to contribute to the development of simple myopia. (Goss, DA; Eskridge JB (1988).

- Degenerative myopia, also known as malignant, pathological, or progressive myopia, is characterized by marked fundus changes, such as posterior staphyloma, and associated with a high refractive error and subnormal visual acuity after correction. This form of myopia gets progressively worse over time. Degenerative myopia has been reported as one of the main causes of visual impairment. Myopia with degenerative changes has been described as being very common in certain races and cultures, such as Chinese, Japanese, Korean, Arab, and Jewish people. (Verma A, Singh D ,2005)

- Nocturnal myopia, also known as night myopia or twilight myopia, is a condition in which the eye has a greater difficulty seeing in low illumination areas, even though its daytime vision is normal. Essentially, the eye's far point of an individual's focus varies with the level of light. Night myopia is believed to be caused by pupils dilating to let more light in, which adds aberrations resulting in becoming more nearsighted. A stronger prescription for myopic night drivers is often needed. Younger people are more likely to be affected by night myopia than the elderly.

- Pseudomyopia is the blurring of distance vision brought about by spasm of the ciliary muscle.

- Induced myopia, also known as acquired myopia, results from exposure to various pharmaceuticals, increases in glucose levels, nuclear sclerosis, or other anomalous conditions The encircling bands used in the repair of retinal detachments may induce myopia by increasing the axial length of the eye. Vukojević, $\mathrm{N}$ 2005)

- Index myopia is attributed to variation in the index of refraction of one or more of the ocular media. Cataracts may lead to index myopia.

- Form deprivation myopia is a type of myopia that occurs when the eye is deprived of clear form vision. Myopia is often induced this way in various animal models to study the pathogenesis and mechanism of myopia development. (Shen, W; Vijayan M, Sivak JG, 2005

- Degree

- Myopia, which is measured in diopters by the strength or optical power of a corrective lens that focuses distant images on the retina, has also been classified by degree or severity.

- Low myopia usually describes myopia of -3.00 diopters or less.

- Medium myopia usually describes myopia between -3.00 and -6.00 diopters. Those with moderate amounts of myopia are more likely to have pigment dispersion syndrome or pigmentary glaucoma.

- High myopia usually describes myopia of -6.00 or more. People with high myopia are more likely to have retinal detachments and primary open angle glaucoma They are also more likely to experience floaters, shadow-like shapes which appear singly or in clusters in the field of vision. Roughly $30 \%$ of myopes have high myopia (Verma A, Singh D. Medicine.com. August 19, 2005).

- Age of onset

- Myopia is sometimes classified by the age of onset

- Congenital myopia, also known as infantile myopia, is present at birth and persists through infancy.

- Youth onset myopia occurs prior to age 20

- School myopia appears during childhood, particularly the school-age years. This form of myopia is attributed to the use of the eyes for close work during the school year (Morgan I, Rose K, 2005).

- Adult onset myopia

- Early adult onset myopia occurs between ages 20 and 40

- Late adult onset myopia occurs after age 40. 

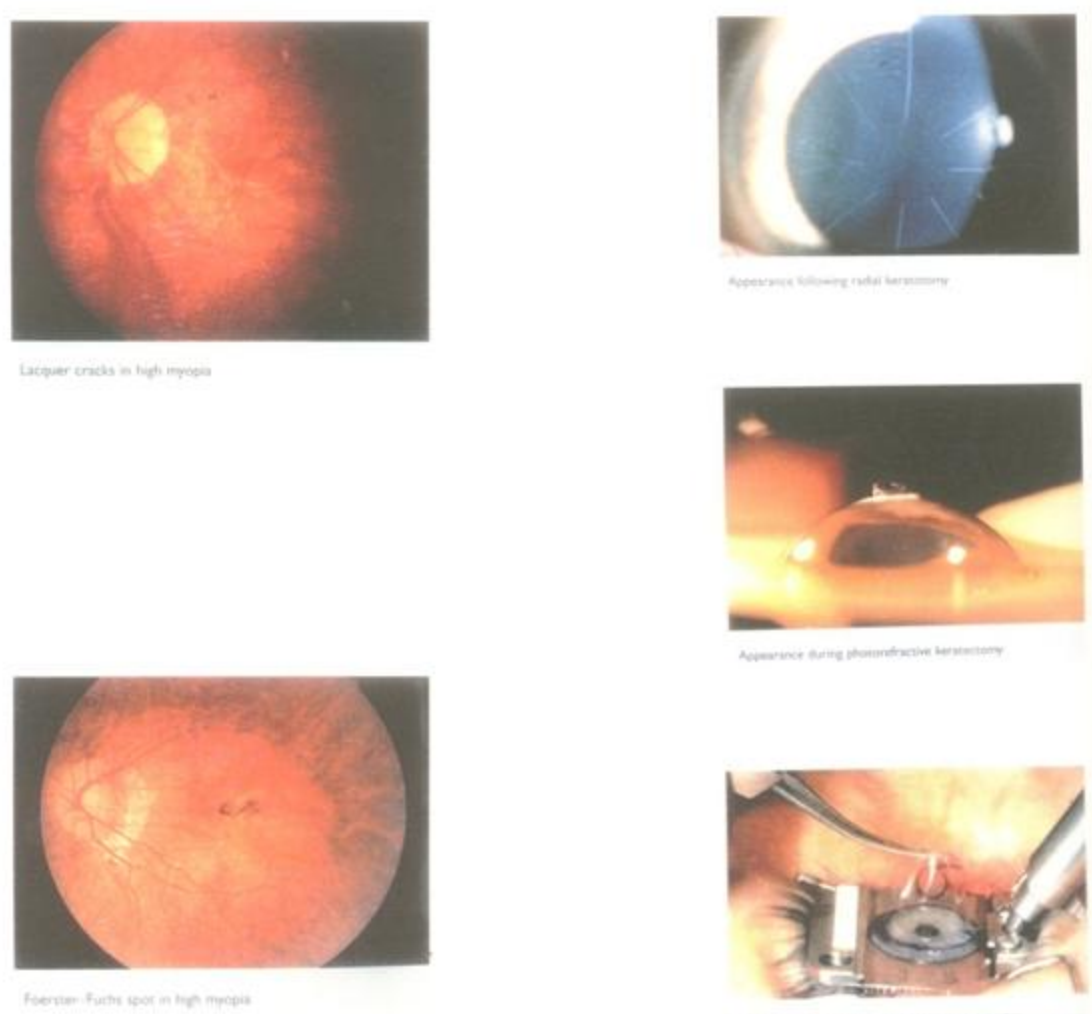

\section{Changes In The Vitreous}

- Changes in the vitreous are almost of constant occurrence in myopia. Myopic degeneration of vitreous closely resembles the senile type. The gel liquefies first at the posterior pole and microfibrillar degeneration seen behind the lens. There will be vitreous opacities which are nothing but aggregation of colloid basis of the gel indicating its disruption without serious complication but they give rise to distressing entoptic opacities.
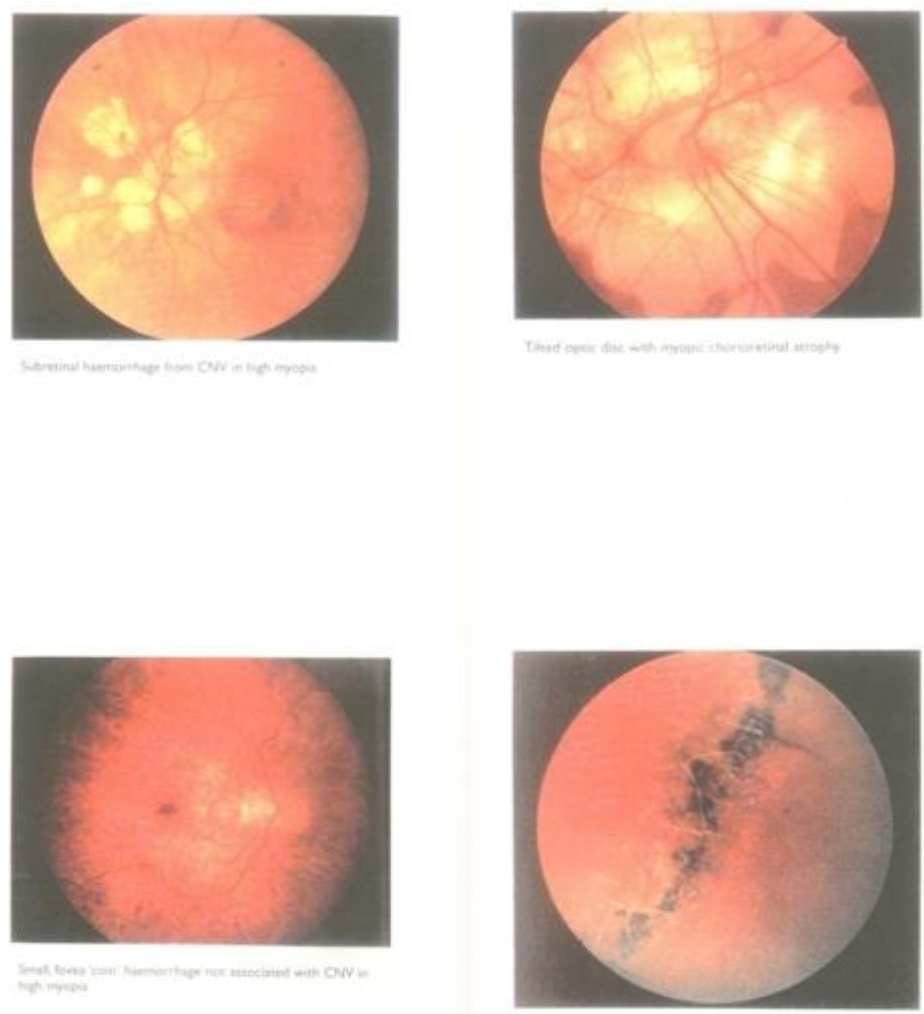

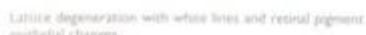




\section{- Changes In The Vitreous}

Changes in the vitreous are almost of constant occurrence in myopia. Myopic degeneration of vitreous closely resembles the senile type. The gel liquefies first at the posterior pole and microfibrillar degeneration seen behind the lens. There will be vitreous opacities which are nothing but aggregation of colloid basis of the gel indicating its disruption without serious complication but they give rise to distressing entoptic opacities

- Changes At The Optic Disc:

- They are frequently the first organic sign to become evident clinically. The myopic crescent usually appears in the first place as a white sharply defined area lying on the temporal side of the optic disc and here the inner surface of the sclera is seen. Some times the sharp edge towards the normal fundus is pigmented and at other times there is a transitional region of brownish red, where the choroid is still partially present and its vessels are seen.

- Supertraction of the retina over the nasal side of the disc

- CHANGES AT THE MACULA:-

- In myopia of pathological type, the earliest recognizable change is irregularity of pigmentation, and a diffuse powdery pigmentation, and the occurrence of small red dots, which are nothing but tufts of dilated capillaries. These macular changes are especially liable to be accompanied by superficial or deep haemorrhages.

Central circular spot: The Forster Fuch's Fleck forms the characteristic feature at the macula. It is due to proliferation of

- CHANGES IN THE SCLERA:

- Scleral ectasias are relatively frequent in the higher degrees of myopia. In the majority of cases the staphyloma involves posterior pole of the eye, but the most common site for sharp crescentic line of shadow is the nasal side of the disc. The sharp shadow indicates an extension nasally of the ectasia at the posterior pole.

- CHANGES IN THE PERIPHERAL RETINA:

- A number of pathological lesions can be seen in the periphery.

- Peripheral cystoid degeneration: Characterized by coarse or fine small cavities which appears separate or some times confluent.

Acquired retinoschisis: Splitting of retina at the outer plexiform layer may be present

- Chorioretinal degeneration: Chorioretinal degeneration usually have an inverse relationship with cystoid degeneration, that is eyes with servere chorioretinal degeneration will have mild cystoid degeneration and vice - versa.

- Pigmentary degeneration: Pigmentary degeneration can be seen in the form of isolated pigment clumps or diffuse or sectorial pigmentation.

- Lattice degeneration: Lattice degeneration can be seen in the form of elongated well demarcated white patches lying circumferentially around the retinal periphery. In well established cases they appear as a network of bluish white lines. The prevalence of lattice degeneration is influenced by the type of axial elongation in high myopia. Retinal breaks will be commonly seen in relation to the lattice lesions.

- Snail track degeneration: Snail track degeneration appears as elongated patches of retinal thinning without any branching white lines, and it may be seen in association with lattice degeneration.

- Retinal breaks: Round holes in relation with lattice degeneration followed by flap tears were the commonest type of retinal breaks causing retinal detachment. The most frequent site being the equatorial region.

\section{- Optics of Myopia}

- The optical condition of myopia is one of where in the dioptric power of the eye is too great for its length or the eye is too long for its dioptric power, so that parallel rays of light come to a focus anterior to the retina, and the image on the retina is made up of the circles of diffusion formed by the diverging beam so that distant objects cannot be seen clearly. 
- Only divergent incident rays will focus on the retina and thus, in order to be seen clearly, an object must be brought close to the eye so that the rays coming from it are rendered sufficiently divergent. The far point (punctumremotum) is, therefore, a finite distance away, and the higher the myopia, the shorter the distance.

- If they are to be brought to a focus at all, parallel rays coming from distant objects must be rendered more divergent and this can be done only by placing a diverging lens infront of the eye.

- To some small extent the myopic compensates for his poor visual acuity, since the nodal point is further away from the retina, the image will be appreciably larger than it would be in the emmetropic eye. With correcting spectacles, however the opposite holds good, and the image appears smaller and brighter.

- Accommodation in Myopia:

- Accommodation is of little value to the myope especially in lower degrees. But in higher degrees of uncorrected myopia, the slightest variation in the distance at which work is done may entail immense accommodative effort if clear vision is to be maintained, so that even if convergence is eliminated and one eye only used, fatigue of accommodation is an important factor in inducing eye strain.

- Diagnosis

A diagnosis of myopia is typically confirmed during an eye examination by an ophthalmologist or an optometrist. Frequently an autorefractor or retinoscope is used to give an initial objective assessment of the refractive status of each eye, then a phoropter is used to subjectively refine the patient's eyeglass prescription

\section{Treatment, management, and prevention}

- Glasses are commonly used to address short-sightedness. Eyeglasses, contact lenses, and refractive surgery are the primary options to treat the visual symptoms of those with myopia. Orthokeratology is the practice of using special rigid contact lenses to flatten the cornea to reduce myopia.

- Eye-exercises and biofeedback

Practitioners and advocates of alternative therapies often recommend eye exercises and relaxation techniques such as the Bates method. However, the efficacy of these practices is disputed by scientists and eye care practitioners. A 2005 review of scientific papers on the subject concluded that there was "no clear scientific evidence" that eye exercises were effective in treating myopia (Rawstron JA,2005

In the eighties and nineties, there was a flurry of interest in biofeedback as a possible treatment for myopia. A 1997 review of this biofeedback research concluded that "controlled studies to validate such methods ... have been rare and contradictory"(G Rupolo,et al,1997). one study that myopes could improve their visual acuity with biofeedback training, but that this improvement was "instrument-specific" and did not generalise to other measures or situations (Randle RJ ., 198

- Prevention

- There is no universally accepted method of preventing myopia. Some clinicians and researchers recommend plus power (convex) lenses in the form of single vision reading lenses or bifocals. A recent Malaysian study reported in New Scientis (Andy Coghlan and Michael Le Page, 2002), suggested that undercorrection of myopia caused more rapid progression of myopia, (Chung K, et al, 2002). Many myopia treatment studies suffer from any of a number of design drawbacks: small numbers, lack of adequate control group, failure to mask examiners from knowledge of treatments used, etc.

- $\quad$ Pirenzepine eyedrops had a limited effect on retarding myopic progression in a recent, placebo-control, double-blinded prospective controlled study ( Siatkowski R, et al, 2004).

- Myopia control

- Various methods have been employed in an attempt to decrease the progression of myopia (Saw SM, et al, 2002). Altering the use of eyeglasses between full-time, part-time, and not at all does not appear to alter myopia progression (Ong E, et al, 1999 and Parssinen O, et al, 1989). Bifocal and progressive lenses have not shown significant differences in altering the progression of myopia (Saw SM, et al, 2002).

\section{Results}

The mean age of the subjects is 20.14 years and maximum affected age group is 16-20 years followed by adult as and adolescents, with a sex ratio of 1: 0.72 (ie. males 58\% ). Family history is Positive only in $21 \%$ of cases. The mean duration of the complaint (DV) in the current sample is $6.07 \pm 0.32$. Child watching the close to TV is the commonest complaint by the parent $50 \% .82 \%$ of the current study consumed diet rich in carbohydrates

- The learning abilities and performance is good in $62 \%$ of the subjects. media is clear in $90 \%$ of the cases. Myopic crescent is noted in $57 \%$ of cases Tsellated fusdus is mpted in $29 \%$ and staphyloma in $2 \%$ of cases.Degeneration is noted in $16 \%$ of the caes. Mean IOP is definitely raised in high myopes (29.06) The mean spherical eqivalent of OD is $-4.21 \pm 0.52$ and of OS is $-4.24 \pm 0.56$. the mean axial length of OD is 
$24.62 \pm 0.21$ and that of OS is $24.44 \pm 0.20$. The meN values for ACD OD is $2.93 \pm 0.05$ and ACD OS is $2.98 \pm 0.07$. The mean corneal Curvare (Vertical) of OD is $7.75 \pm 0.03$ and of OS is $7.73 \pm 0.03$

- Strong (-)ve correlation is noticed between axial lenh and spherical equivalent of refraction (-0.73) and moderate correaltion between ACD, Coneal curvature and sphereical equivalent of refraction. Linear Regression analysis of Axial length vs sphericl equivalent of refraction of BE revealed ANOVA as 0.0002 , significant at $98 \mathrm{df}$ and regression coeeficient (Beta value) being 0.85 .

- From a research perspective, the results from this clinical trial, shed light on mechanisms of myopia and will help to identify risk factors related to myopia in children.

\section{Conclusion}

1. The present study shows that the adolescent age group forms the high risk group for refractive errors and most of the children are unaware of refractive errors.

2. Due emphasis must, therefore, be laid upon the screening for refractive errors in this age group.

3. Moreover, attention should be paid to visual hygiene. While reading, illumination should be good and adequately arranged.

4. Posture should be easy and natural. Clarity and print should be carefully supervised.

5. An undue ocular fatigue should be avoided. Students should have as many outdoor hours as possible.

6. Public need to be educated about signs and symptoms of refractive errors.

7. Pre-school and school screening for detection of refractive errors should be carried out on periodic basis.

8. Longitudinal studies to further assess our findings are warranted

9. Although small difference is greater than what would be expected by chance alone, from a clinical perspective it is not large enough to recommend a change in the way eye care professionals prescribe glasses for children with myopia.

10. There is a paucity of studies on intervention in myopia progression in children as they are difficult to carry out. It is hoped that a better understanding of myopia progression will lead to easier methods for carrying out such studies

\section{Limitations}

- The sample size is small

- Only cases attending Hospital were recruited for the study.

\section{References}

[1]. A J Lee,et al 2004A J Lee, S-M Saw, G Gazzard, A Cheng D T H Tan ,2004,Intraocular pressure associations with refractive error and axial length in children British Journal of Ophthalmology 2004;88:5-7

[2]. Bayramlar H, Cekic O, Hepsen IF 1999"Does convergence, not accommodation, cause axial-length elongation at near? A biometric study in teens." Ophthalmic Res. 1999;31(4):304-8

[3]. Edwards MH, et al 1963 Chun CY, Leung SS. Intraocular pressure in an unselected sample of 6- to 7- year-old Chinese children. Optom Vis Sci 1993;70:198-200

[4]. Hammond CJ, et al 2004 T, Mak YT, Spector TD. "A susceptibility locus for myopia in the normal population is linked to the PAX6 gene region on chromosome 11: a genomewide scan of dizygotic twins." Am J Hum Genet. 2004 Aug;75(2):294-304. Epub 2004 Jun 24

[5]. IS Jain, et al1983 Sandeep Jain, Kanwar Mohan,1983 The epidemiology of high myopia-chanding trends; Ind J Oph Year : 1983 | Volume : 31 | Issue : 6 | Page : 723-728 